\title{
Hanbury Brown-Twiss effect and thermal light ghost imaging: A unified approach
}

\author{
Li-Gang Wang, ${ }^{1,2}$ Sajid Qamar, ${ }^{3}$ Shi-Yao Zhu, ${ }^{1,2}$ and M. Suhail Zubairy ${ }^{3,4}$ \\ ${ }^{1}$ Department of Physics, Zhejiang University, 310027 Hangzhou, China \\ ${ }^{2}$ Department of Physics, Chinese University of Hong Kong, Shatin, N. T., Hong Kong \\ ${ }^{3}$ Center for Quantum Physics, COMSATS Institute of Information Technology, Islamabad, Pakistan \\ ${ }^{4}$ Department of Physics and Institute for Quantum Studies, Texas A\&M University, College Station, Texas 77843-4242, USA
}

(Received 14 September 2008; revised manuscript received 1 January 2009; published 24 March 2009)

\begin{abstract}
We compare the Hanbury Brown-Twiss (HBT) and the thermal light ghost imaging schemes in both near and far fields. Both effects arise as a result of the intensity fluctuations of the thermal light and we find that the essential physics behind the two effects is the same. The difference however is that, in the ghost imaging, large number of bits information of an object needs to be treated together, whereas, in the HBT, there is only one bit information required to be obtained. In the HBT experiment far field is used for the purpose of easy detection, while in the ghost image experiment near (or not far) field is used for good quality image.
\end{abstract}

DOI: 10.1103/PhysRevA.79.033835

PACS number(s): 42.50.Ar, 42.50.Dv, 42.50.St

Recently, there is a heated discussion on the physics of the ghost imaging (GI) with thermal light [1-5]. For example, in a recent paper Scarcelli et al. [5] pose the question: "Can two-photon correlation of chaotic light be considered as correlation of intensity fluctuations?" The authors point out that near field is required for ghost imaging whereas the far field is required in the Hanbury Brown-Twiss (HBT) experiment. They also conclude that the chaotic (thermal) light ghost imaging could not be explained with classical mechanics, and the physics of the ghost imaging is not the intensity fluctuations of the thermal light $\left\langle\Delta I_{1} \Delta I_{2}\right\rangle=\left\langle I_{1} I_{2}\right\rangle-\left\langle I_{1}\right\rangle\left\langle I_{2}\right\rangle$ as in the HBT experiment [2,5], because large size of the source (near field) results in $\left\langle\Delta I_{1} \Delta I_{2}\right\rangle=0$ [2]. Instead, they claim that the essential physics is the two-photon quantum interference $[2,5]$. On the other hand, others disagreed with the conclusions in [5] by Shih et al. [3,4], and have concluded that the ghost imaging with classical thermal field is essentially a classical effect $[3,4]$. The controversy has persisted by another paper of Shih's group [2] in reply to comments in [4]. The main objective of this paper is to resolve this controversy. In particular, we carry out a simple analysis that helps to clarify the difference and similarity between the GI and HBT, and hence shed light on the physics behind the two related effects.

Originally the ghost image was achieved with entangled light [6]. In 2004 the formation of ghost image with thermal light was predicted [7] and the equation for the image formation is given in Ref. [8]. In 2005 the experiments on ghost imaging with thermal light were realized [9-11]. Since then, theoretical models are put forward to explain the thermal light ghost imaging $[3,12,13]$. Until today, far field is used in the HBT experiments, while near field (not-far field) is used in the ghost imaging experiments [2,5]. There is a first-order coherence for the far field, while the first-order coherence for the near field (not-far field) is small. A question of interest is whether the far field and the near field result in significantly different physics. We ask ourselves, what will be the results if we use the far field and the near field for both HBT and GI experiments. In the present paper, we address this question and discuss how the answer to this question reveals the physics behind the GI and the HBT effect.

The setup for the thermal light ghost imaging experiment is presented in Fig. 1 with a lens immediately behind the object focusing onto $\mathrm{D}_{2}$ (as a bucket detector [5]). The simplest HBT experiment $[5,14]$ is the same except the detector $\mathrm{D}_{2}$ is placed at the location of the object with no object and lens. In both experiments, the source is a surface thermal light, for example a black box at a certain temperature. The thermal light from the source is split by a beam splitter and shines the two detectors, $D_{1}$ and $D_{2}$ through two paths, as shown in Fig. 1. The second-order correlation is detected by $\mathrm{D}_{1}$ and $\mathrm{D}_{2}$ in both the experiments. For the thermal light, the field statistics is Gaussian and this allows the calculation of the second-order correlation from the first-order correlation, i.e.,

$$
\mathrm{G}^{2}\left(u_{1}, u_{2}\right)=\left\langle I_{1} I_{2}\right\rangle=\left\langle I\left(u_{1}\right)\right\rangle\left\langle I\left(u_{2}\right)\right\rangle+\left|\Gamma\left(u_{1}, u_{2}\right)\right|^{2},
$$

where $\left\langle I\left(u_{1,2}\right)\right\rangle$ are the intensities at the points $u_{1}$ and $u_{2}$ on the planes of $\mathrm{D}_{1}$ and $\mathrm{D}_{2}$, and $\Gamma\left(u_{1}, u_{2}\right)=\left\langle E^{+}\left(u_{1}\right) E\left(u_{2}\right)\right\rangle$ is the cross correlation. The correlations at the detectors can be found out from the correlation at the source by correlation propagation method [14].

$$
\begin{aligned}
I\left(u_{1,2}\right) & =\left\langle E^{+}\left(u_{1,2}\right) E\left(u_{1,2}\right)\right\rangle \\
& =\iint\left\langle E_{s}^{+}\left(x_{1}\right) E_{s}\left(x_{2}\right)\right\rangle h_{1,2}^{*}\left(x_{1}, u_{1,2}\right) h_{1,2}\left(x_{2}, u_{1,2}\right) d x_{1} d x_{2},
\end{aligned}
$$

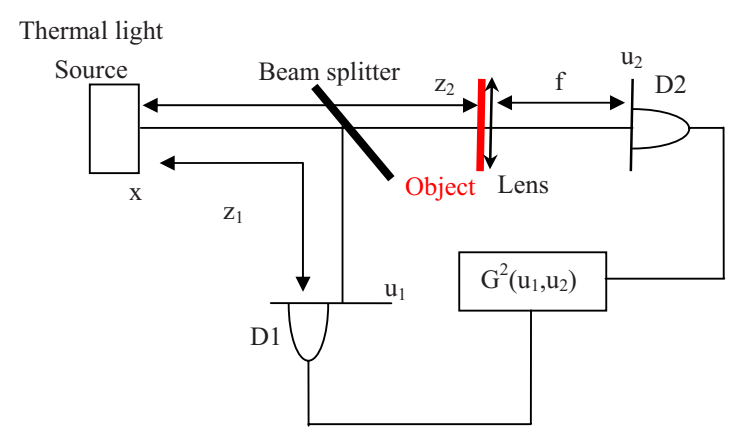

FIG. 1. (Color online) Ghost image setup. 


$$
\begin{aligned}
\Gamma\left(u_{1}, u_{2}\right) & =\left\langle E^{+}\left(u_{1}\right) E\left(u_{2}\right)\right\rangle \\
& =\iint\left\langle E_{s}^{+}\left(x_{1}\right) E_{s}\left(x_{2}\right)\right\rangle h_{1}^{*}\left(x_{1}, u_{1}\right) h_{2}\left(x_{2}, u_{2}\right) d x_{1} d x_{2},
\end{aligned}
$$

where $x_{1,2}$ are the points at the source plane and the integrals are within the source. Here $h_{1,2}\left(x, u_{1,2}\right)$ are the propagation functions of the correlation from the source to the detectors along paths 1 and 2, respectively, which depend on the optical elements in the paths. For path $1, h_{1}\left(x, u_{1}\right)$ is the same in the two experiments.

$$
h_{1}^{\mathrm{H}, \mathrm{G}}\left(x, u_{1}\right)=\left(-\frac{i}{\lambda z_{1}}\right)^{1 / 2} \exp \left[-\frac{i \pi}{\lambda z_{1}}\left(x^{2}-2 x u_{1}+u_{1}^{2}\right)\right] .
$$

For path 2 , we have different $h_{2}\left(x, u_{2}\right)$ for the two experiments,

$$
\begin{aligned}
h_{2}^{\mathrm{H}}\left(x, u_{2}\right) & =\left(-\frac{i}{\lambda z_{2}}\right)^{1 / 2} \exp \left[-\frac{i \pi}{\lambda z_{2}}\left(x^{2}-2 x u_{2}+u_{2}^{2}\right)\right], \\
h_{2}^{\mathrm{G}}\left(x, u_{2}\right)= & \left(-\frac{i}{\lambda f}\right)^{1 / 2}\left(-\frac{i}{\lambda z_{2}}\right)^{1 / 2} \int d v H(v) \\
& \times \exp \left[-\frac{i \pi}{\lambda z_{2}}\left(x^{2}-2 x v+v^{2}\right)-\frac{i \pi}{\lambda f}\left(-2 v u_{2}+u_{2}^{2}\right)\right],
\end{aligned}
$$

where the superscripts $\mathrm{H}$ and $\mathrm{G}$ indicate $\mathrm{HBT}$ and GI, respectively. It is implicit that $h_{1,2}^{\mathrm{H}, \mathrm{G}}\left(x, u_{1,2}\right)$ depend also on $z_{1}$ or $z_{2}$. In Eq. (5b), $H(v)$ is the transmittance of the object and the integration is due to the bucket detector. In general, we should consider the two-dimensional imaging. However as $x$ and $y$ directions are independent, we only consider the $x$ direction (one dimension). This however does not affect the physics.

For the thermal light at the source, the first-order correlation can be written as a series of the form $[15,16]$,

$$
\left\langle E_{s}^{+}\left(x_{1}\right) E_{s}\left(x_{2}\right)\right\rangle \propto 1-\alpha\left(x_{1}-x_{2}\right)^{2}+\beta\left(x_{1}-x_{2}\right)^{4}+\cdots
$$

with $\beta / \alpha=2.2$. This however does not allow for an analytical solution for the correlation functions of the field. We therefore approximate the first-order correlation function of the source to be a Gaussian Schell model source $[15,17]$,

$$
\left\langle E_{s}^{+}\left(x_{1}\right) E_{s}\left(x_{2}\right)\right\rangle=G_{0} \exp \left[-\frac{x_{1}^{2}+x_{2}^{2}}{4 \sigma_{I}^{2}}-\frac{\left(x_{1}-x_{2}\right)^{2}}{2 \sigma_{g}^{2}}\right] .
$$

Here we have a Gaussian distribution for the intensity of the source with the width $\sigma_{I}$ and $\sigma_{g}$ is the first-order transverse coherence width (correlation length) of the thermal light source. The normalized second-order correlations (HBT or GI) for the two experiments are

$$
\text { HBT or } \operatorname{GI}\left(u_{1}, u_{2}, z_{1}, z_{2}\right)=\frac{\left|\Gamma\left(u_{1}, u_{2}\right)\right|^{2}}{\left(\left\langle I\left(u_{1}\right)\right\rangle\left\langle I\left(u_{2}\right)\right\rangle\right)} .
$$

First we consider the HBT experiment. The point detectors are located at $u_{1} \neq 0$ and $u_{2}=0$ (transverse HBT). From

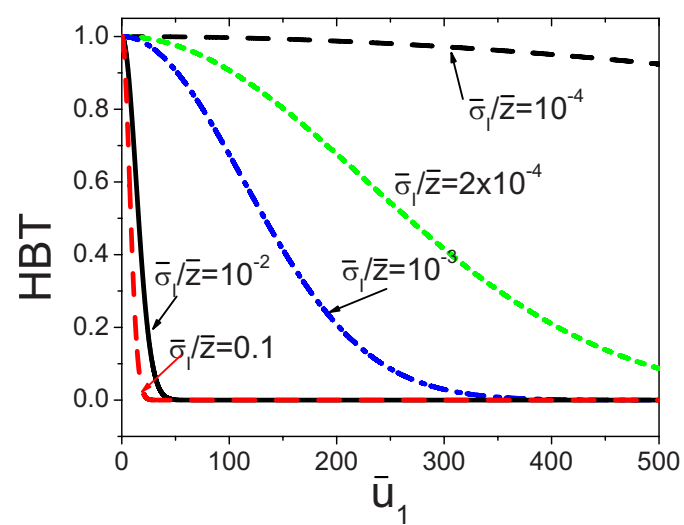

FIG. 2. (Color online) HBT effect for different $\bar{\sigma}_{I} / \bar{z}=10^{-4}$, $2 \times 10^{-4}, 10^{-3}, 10^{-2}$, and 0.1 (from top to below), with $\bar{\sigma}_{g}=10$ and $\bar{z}=10^{5}$.

Eqs. (2)-(5) with $z_{1}=z_{2}=z$ we have $\left|\Gamma\left(\bar{u}_{1}, 0\right)\right|^{2}=\frac{\left(4 \pi G_{0}\right)^{2}}{\bar{\xi}}$ $\times \exp \left\{-\frac{8 \pi^{2}\left(\bar{\sigma}_{g}^{2}+2 \bar{\sigma}_{T}^{2}\right) \bar{u}_{1}^{2}}{\bar{\sigma}_{g}^{2} \bar{\sigma}_{T}^{2} \bar{\xi}}\right\}$, and $\left\langle I\left(\bar{u}_{1,2}\right)\right\rangle=\frac{4 \pi G_{0}}{\bar{\xi}^{1 / 2}} \exp \left\{-\frac{8 \pi^{2} \bar{u}_{1,2}^{2}}{\bar{\xi}_{I}^{2}}\right\}$, which are substituted into Eq. (8) we obtain,

$$
\operatorname{HBT}\left(\bar{u}_{1}, 0\right)=\exp \left[-\frac{\bar{u}_{1}^{2}}{\bar{\sigma}_{g}^{2}+\left(\bar{z}^{2} / 4 \pi^{2} \bar{\sigma}_{I}^{2}\right)\left(\bar{\sigma}_{g}^{2} / 4 \bar{\sigma}_{I}^{2}+1\right)}\right],
$$

where $\bar{u}_{1}^{2}, \bar{z}$, and $\bar{\sigma}_{I, g}$ are in units of $\lambda$. If $\bar{u}_{1}=\bar{u}_{2}=0$, we have HBT $=1$. As $\bar{u}_{1}^{2}$ increases, HBT decreases. In Fig. 2 , we plot HBT versus $\bar{u}_{1}$ with $\bar{\sigma}_{g}=10$ and $\bar{z}=10^{5}$ for $\bar{\sigma}_{I} / \bar{z}=10^{-4}$, $2 \times 10^{-4}, 10^{-3}, 10^{-2}$, and 0.1 . In the following, we define $\bar{\sigma}_{I} / \bar{z}<0.05$ as the far field, and otherwise as the near field.

In the experiment with thermal light, the condition $\bar{z}, \bar{\sigma}_{I}^{2}$ $\gg \bar{\sigma}_{g}^{2}$ is always satisfied. We can therefore rewrite Eq. (9) as

$$
\operatorname{HBT}\left(\bar{u}_{1}, 0\right)=\exp \left(-\frac{\bar{u}_{1}^{2}}{\bar{\sigma}_{g}^{2}+\frac{\bar{z}^{2}}{4 \pi^{2} \bar{\sigma}_{I}^{2}}}\right),
$$

which is a Gaussian distribution. For small $\bar{\sigma}_{I} / \bar{z}$ (the far field), the decrease in HBT with the increase in $\bar{u}_{1}$ is slow; see Fig. 2. That is to say, the smaller $\bar{\sigma}_{I} / \bar{z}$ is, the easier the HBT can be measured experimentally. This is why the HBT experiment is usually done in the far field. However, the HBT experiment can be carried out in principle with the near field (or not-far field), because at $\bar{u}_{1}=0$ we always have HBT $=1$, no matter what is the values of $\bar{\sigma}_{I}, \bar{z}$, and $\bar{\sigma}_{g}$. Large $\bar{\sigma}_{I} / \bar{z}$ (near field or not-far field) does not change the physical nature of the HBT experiment (the intensity fluctuations), but it increases the difficulty to realize the HBT experiment.

For the GI experiment, it follows on substituting Eqs. (4) and (5b) into Eq. (3) and with $z_{1}=z_{2}$ where the image is formed, we obtain the cross correlation function and the intensity, 


$$
\begin{aligned}
\Gamma\left(u_{1}, 0\right)= & \frac{4 \pi G_{0} \exp \left(\frac{i \pi}{\bar{z}_{1}} \bar{u}_{1}^{2}\right)}{\bar{f}^{1 / 2} \bar{\xi}^{1 / 2}} \int d \bar{v} H(\bar{v}) \exp \left(-\frac{i \pi}{\bar{z}_{1}} \bar{v}^{2}\right) \\
& \times \exp \left\{-\frac{4 \pi^{2}\left[\left(\bar{\sigma}_{g}^{2}+2 \bar{\sigma}_{I}^{2}\right) \bar{u}_{1}^{2}-4 \bar{\sigma}_{I}^{2} \bar{u}_{1} \bar{v}+\left(\bar{\sigma}_{g}^{2}+2 \bar{\sigma}_{I}^{2}\right) \bar{v}^{2}-i 4 \pi \bar{\sigma}_{g}^{2} \bar{\sigma}_{I}^{2}\left(\bar{v}^{2}-\bar{u}_{1}^{2}\right) / \bar{z}_{1}\right]}{\bar{\sigma}_{g}^{2} \bar{\sigma}_{I}^{2} \bar{\xi}}\right\}, \\
\left\langle I\left(u_{2}=0\right)\right\rangle= & \left.\frac{4 \pi G_{0} \iint d \bar{v}_{1} d \bar{v}_{2} H\left(\bar{v}_{1}\right) H\left(\bar{v}_{2}\right) \exp \left(-\frac{i \pi}{\bar{z}_{1}} \bar{v}_{2}^{2}+\frac{i \pi}{\bar{z}_{1}^{2}} \bar{v}_{1}^{2}\right)}{\bar{\xi}^{1 / 2}}\right\} \\
& \times \exp \left\{-\frac{4 \pi^{2}\left[\left(\bar{\sigma}_{g}^{2}+2 \bar{\sigma}_{I}^{2}\right) \bar{v}_{1}^{2}-4 \bar{\sigma}_{I}^{2} \bar{v}_{1} \bar{v}_{2}+\left(\bar{\sigma}_{g}^{2}+2 \bar{\sigma}_{I}^{2}\right) \bar{v}_{2}^{2}-i 4 \pi \bar{\sigma}_{g}^{2} \bar{\sigma}_{I}^{2}\left(\bar{v}_{2}^{2}-\bar{v}_{1}^{2}\right) / \bar{z}_{1}\right]}{\bar{\sigma}_{g}^{2} \bar{\sigma}_{I}^{2} \bar{\xi}}\right\},
\end{aligned}
$$

and $\left\langle I\left(\bar{u}_{1}\right)\right\rangle=\left(4 \pi G_{0} / \bar{\xi}^{1 / 2}\right) \exp \left[-8 \pi^{2} \bar{u}_{1}^{2} / \bar{\sigma}_{I}^{2} \bar{\xi}\right]$, where $\bar{\xi}=16 \pi^{2}$ $+\left(\bar{z}_{1}^{2} / \bar{\sigma}_{I}^{2}\right)\left(4 / \bar{\sigma}_{g}^{2}+1 / \bar{\sigma}_{I}^{2}\right)$. With Eqs. (10a) and $(10 \mathrm{~b})$, we calculate $\operatorname{GI}\left(\bar{u}_{1}, \bar{z}_{1}=\bar{z}_{2}\right)=\left|\Gamma\left(\bar{u}_{1}\right)\right|^{2} /\left\langle I\left(\bar{u}_{1}\right)\right\rangle\left\langle I\left(\bar{u}_{2}=0\right)\right\rangle$ numerically. In Fig. 3, we plot the ghost image for a triple slits object (with the width of each slit being $10 \lambda$ and the separation between the two slits being $10 \lambda$ ) with $\bar{z}_{1}=\bar{z}_{2}=10^{5}$ for different values of $\bar{\sigma}_{I}$ and $\bar{\sigma}_{g}$. Within the three slits, $H(\bar{v})=1,0.8$, and 0.6 , respectively, and is zero elsewhere.

From Fig. 3, we see no image for small $\bar{\sigma}_{1} / \bar{z}_{1}$ (far field) [curves (a) and (b)]. Note that decreasing $\bar{\sigma}_{g}$ does not help for small $\bar{\sigma}_{1} / \bar{z}_{1}$. For a good quality image we need large $\bar{\sigma}_{1} / \bar{z}_{1}$ (near field) $[18,19]$ and small $\bar{\sigma}_{g}$. In curves (d) and (e), we note the formation of the image and the image edge of the middle slit spreads approximately from 3 to 7 and 4 to 6 (see the inset) with visibilities of $12 \%$ and $7 \%$, respectively. When we have good quality image, the visibility is low which is in agreement with earlier studies [8,20,21]. Largesize slits result in low visibility.

For small $\bar{\sigma}_{I} / \bar{z}_{1} \ll 1$ (far field) we have HBT effect, but no thermal light ghost image. The difference can be explained as follows. In the HBT experiment, the measurement mainly differentiates between two values, HBT $=1$ or 0 . This corresponds to one bit information. On the other hand, in the

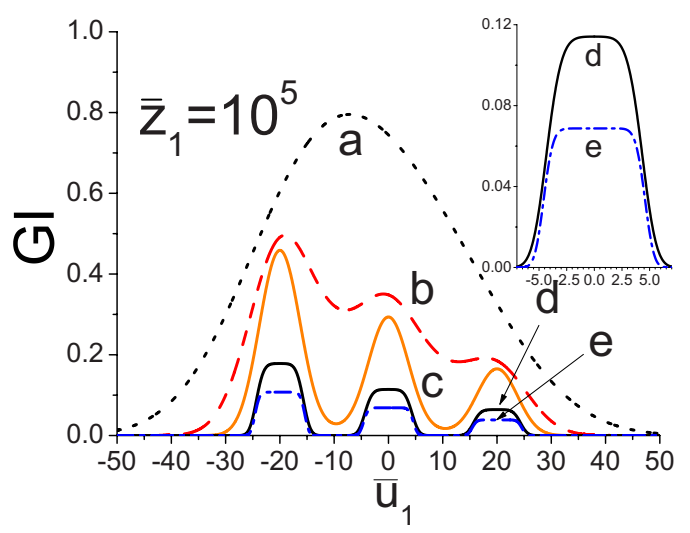

FIG. 3. (Color online) The ghost image of three slits, (a) $\bar{\sigma}_{I} / \bar{z}_{1}=10^{-2}$, (b) $\bar{\sigma}_{I} / \bar{z}_{1}=2.5 \times 10^{-2}$, (c) $\bar{\sigma}_{I} / \bar{z}_{1}=0.2$, with $\bar{\sigma}_{g}=4$, (d) $\bar{\sigma}_{I} / \bar{z}_{1}=0.2$ and $\bar{\sigma}_{g}=1$, and (e) $\bar{\sigma}_{I} / \bar{z}_{1}=0.2$ and $\bar{\sigma}_{g}=0.1$. ghost image, we need to obtain the information of the whole object and this corresponds to a large amount of bits. The large amount of bits is processed together and one particular bit must not be influenced by other bits. For the curve (c) in Fig. 3, it is hard to say whether the image is formed. If we consider the three slits as three bits, we can conclude: "yes, we have three bits with good visibility in curve (c)."

Let us consider a very narrow slit for the object located at $\bar{v}=\bar{a}$. The image measurement becomes the determination of one nonzero value at one location (and near by) and zero value at other locations, which is equivalent to the measurement in the HBT experiment to obtain one bit information. Setting $\bar{z}_{1}, \bar{\sigma}_{I} \gg \bar{\sigma}_{g}$ (valid in experiments) we have

$$
\frac{\left|\Gamma\left(\bar{u}_{1}, \bar{u}_{2}=0\right)\right|^{2}}{\left\langle I\left(\bar{u}_{1}\right)\right\rangle\left\langle I\left(\bar{u}_{2}=0\right)\right\rangle}=\exp \left[-\frac{\left(\bar{u}_{1}-\bar{a}\right)^{2}}{\bar{\sigma}_{g}^{2}+\left(\bar{z}_{1}^{2} / 4 \pi^{2} \bar{\sigma}_{I}^{2}\right)}\right] .
$$

The image of the very narrow slit is a Gaussian distribution with a width of $\left\{\bar{\sigma}_{g}^{2}+\left(\bar{z}_{1}^{2} / 4 \pi^{2} \bar{\sigma}_{I}^{2}\right)\right\}^{1 / 2}$ [the same as Eq. (9a) for the HBT effect]. The width of the best image for an ideal point at $\bar{u}_{20}$ is limited by the width $\left\{\bar{\sigma}_{g}^{2}+\left(\bar{z}_{1}^{2} / 4 \pi^{2} \bar{\sigma}_{I}^{2}\right)\right\}^{1 / 2}$. For small $\bar{\sigma}_{I} / \bar{z}_{1}(\ll 1$, far field), the ghost image of the very narrow slit can still be formed but with very bad quality (wide spread). This is the reason that the good image for small $\bar{\sigma}_{I} / \bar{z}_{1}$ (far field) cannot be achieved even in the limit of $\bar{\sigma}_{g}$ $\rightarrow 0$. While for a large $\bar{\sigma}_{I} / \bar{z}_{1}\left(=0.2\right.$, not far field), small $\bar{\sigma}_{g}$ may lead to small width (good image quality), a similar situation for the HBT experiment.

The accuracy of the image is limited by the width of the detector (no real point detector). Consequently, improving the quality of the image by increasing the size source is limited by the width of the detector. Also the width determines the possibility of detecting the HBT with large size of source, the width $\leq \lambda\left\{\bar{\sigma}_{g}^{2}+\left(\bar{z}_{1}^{2} / 4 \pi^{2} \bar{\sigma}_{I}^{2}\right)\right\}^{1 / 2}$ [see Eq. (9a)]. Within this limit, any size in the object equal to the width represents one bit. Currently, the width of the best detector is about 10 $\lambda$. In Fig. 4, we plot the images of objects contained one bit, two bits, and three bits for the detector with a width of $8 \lambda$. For one bit object, the visibility is almost one, for the two bits and three bits objects, the visibility reduce to half 


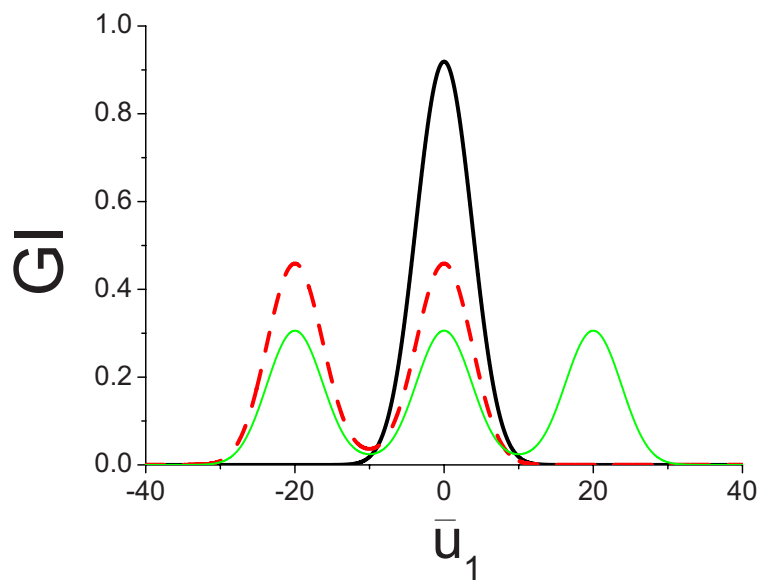

FIG. 4. (Color online) The visibility of the objects containing one bit, two bits, and three bits information. The width of each slit and the interval between slits are $10 \lambda$, and $\bar{\sigma}_{I} / \bar{z}_{1}=0.2, \bar{\sigma}_{g}=4$.

and one-third. The more information in the object, the lower the image visibility will be.

Let us consider $\bar{\sigma}_{g} \rightarrow 0$ and $\bar{\sigma}_{I} / \bar{z} \gg 1$. Under this limit, we have $\mathrm{HBT}=1$ at $\bar{u}_{1}=0$, and $\mathrm{HBT} \approx 0$ for $\bar{u}_{1} \neq 0$, which can be considered as perfect bunching (photoelectrons always comes out in pairs). It can be proven from Eqs. (10) that under this limit we have $\Gamma\left(\bar{u}_{1}, \bar{u}_{2}=0\right) \propto H\left(\bar{u}_{1}\right)$ [13] (perfect image). Therefore, we can say the perfect bunching results in the perfect image. Under this limit, we can prove

$$
\left|\Gamma\left(\bar{u}_{1}, \bar{u}_{2}=0\right)\right|^{2} /\left\langle I\left(\bar{u}_{2}=0\right)\right\rangle\left\langle I\left(\bar{u}_{1}\right)\right\rangle=\left|H\left(\bar{u}_{1}\right)\right|^{2} / \int d \bar{v}|H(\bar{v})|^{2},
$$

perfect imaging with the visibility inversely proportional to the size of the object (not the size of the source). Large-size source improves the quality of the image, and large-size object reduces the visibility, which comes from the bucket detection triggered by the thermal light passing through different points of the object [20]. Here we would like to emphasize that large size of the source does not result in the low visibility as claimed in [5], and it is the size of the object that leads to low visibility.

Large size of the source results in good quality image, while large size of the object (a large amount of bits) leads to low visibility. Therefore we can conclude that the physics behind the thermal light ghost imaging and the HBT experiment is the same, the intensity fluctuations. The difference between the GI and the HBT experiments is the information that is required to be obtained: large amount for GI and a small amount for HBT (large number of bits versus one bit). In the HBT experiment the far field is used for the purpose of easy detection, while in the GI experiment the near field (not-far field) is used for good quality image at the expense of low visibility [22]

This work is supported by HKUST3/06C of HK Government, FRG of HKBU, and NSFC (Contract No. 10604047). The research of MSZ is supported by a grant from Qatar National Research Fund (QNRF).

\section{APPENDIX: DERIVATION OF EOS. (11) and (12)}

Now let us see how to obtain Eq. (11). Since we assume a very narrow slit at position $\bar{v}=\bar{a}$, then the object function could be written by $H(\bar{v})=\delta(\bar{v}-\bar{a})$. From Eq. (10a), we can easily find the cross correlation,

$$
\left|\Gamma\left(\bar{u}_{1}, 0\right)\right|^{2}=\frac{\left(4 \pi G_{0}\right)^{2}}{\overline{f \xi}} \exp \left\{-\frac{8 \pi^{2}\left[\left(\bar{\sigma}_{g}^{2}+2 \bar{\sigma}_{I}^{2}\right) \bar{u}_{1}^{2}-4 \bar{\sigma}_{I}^{2} \bar{u}_{1} \bar{a}+\left(\bar{\sigma}_{g}^{2}+2 \bar{\sigma}_{I}^{2}\right) \bar{a}^{2}\right]}{\bar{\sigma}_{g}^{2} \bar{\sigma}_{I}^{2} \bar{\xi}}\right\} .
$$

Meanwhile, from Eq. (10b) the intensity at $u_{2}=0$ in the GI experiment could be obtained

$$
\left\langle I\left(\bar{u}_{2}=0\right)\right\rangle=\frac{4 \pi G_{0}}{\bar{f} \bar{\xi}^{1 / 2}} \exp \left\{-\frac{4 \pi^{2}\left[\left(\bar{\sigma}_{g}^{2}+2 \bar{\sigma}_{I}^{2}\right) \bar{a}^{2}-4 \bar{\sigma}_{I}^{2} \bar{a}^{2}+\left(\bar{\sigma}_{g}^{2}+2 \bar{\sigma}_{I}^{2}\right) \bar{a}^{2}\right]}{\bar{\sigma}_{g}^{2} \bar{\sigma}_{I}^{2} \bar{\xi}}\right\} .
$$

From Eq. (8), we finally obtain

$$
\operatorname{GI}\left(\bar{u}_{1}, \bar{u}_{2}=0\right)=\frac{\left|\Gamma\left(\bar{u}_{1}, 0\right)\right|^{2}}{\left\langle I\left(\bar{u}_{1}\right)\right\rangle\left\langle I\left(\bar{u}_{2}=0\right)\right\rangle}=\exp \left[-\frac{\left(\bar{u}_{1}-\bar{a}\right)^{2}}{\bar{\sigma}_{g}^{2}+\frac{\bar{z}_{1}^{2}}{4 \pi^{2} \bar{\sigma}_{I}^{2}}\left(1+\frac{\bar{\sigma}_{g}^{2}}{4 \bar{\sigma}_{I}^{2}}\right)}\right] .
$$

It is clearly seen that Eq. (A3) is similar to Eq. (9), which indicates that the image of an ideal pointlike object in GI experiment actually becomes a Gaussian-shape image, and the image size is limited by the propagation distance $z_{1}$, the spatial coherence $\bar{\sigma}_{g}$, and the light source size $\bar{\sigma}_{I}$. Under the conditions of $\bar{z}_{1}, \bar{\sigma}_{I} \gg \bar{\sigma}_{g}$ (valid in experiments), Eq.(A3) could be approximately expressed by 


$$
\operatorname{GI}\left(\bar{u}_{1}, \bar{u}_{2}=0\right)=\frac{\left|\Gamma\left(\bar{u}_{1}, 0\right)\right|^{2}}{\left\langle I\left(\bar{u}_{1}\right)\right\rangle\left\langle I\left(\bar{u}_{2}=0\right)\right\rangle}=\exp \left[-\frac{\left(\bar{u}_{1}-\bar{a}\right)^{2}}{\bar{\sigma}_{g}^{2}+\frac{\bar{z}_{1}^{2}}{4 \pi^{2} \bar{\sigma}_{I}^{2}}}\right],
$$

which is Eq. (11).

In order to prove Eq. (12), we can rewrite Eqs. (10a) and (10b) as follows:

$$
\begin{aligned}
& \Gamma\left(u_{1}, 0\right)=\frac{4 \pi G_{0}}{\bar{f}^{1 / 2} \bar{\xi}^{1 / 2}} \int d \bar{v} H(\bar{v}) \exp \left(\frac{i \pi}{\bar{z}_{1}} \bar{u}_{1}^{2}-\frac{i \pi}{\bar{z}_{1}} \bar{v}^{2}\right) \exp \left\{-\frac{4 \pi^{2}\left[\left(\bar{\sigma}_{g}^{2}+2 \bar{\sigma}_{I}^{2}\right) \bar{u}_{1}^{2}-4 \bar{\sigma}_{I}^{2} \bar{u}_{1} \bar{v}+\left(\bar{\sigma}_{g}^{2}+2 \bar{\sigma}_{I}^{2}\right) \bar{v}^{2}\right]}{\bar{\sigma}_{g}^{2} \bar{\sigma}_{I}^{2} \bar{\xi}}+\frac{i 16 \pi^{3}\left(\bar{v}^{2}-\bar{u}_{1}^{2}\right)}{\bar{\xi}_{1}}\right\} \\
& \stackrel{\bar{z} / \bar{\sigma}_{I} \rightarrow 0}{\rightarrow} \frac{4 \pi G_{0}}{\bar{f}^{1 / 2} \bar{\xi}^{1 / 2}} \int d \bar{v} H(\bar{v}) \exp \left\{-\frac{1}{4}\left[\left(\frac{1}{\bar{\sigma}_{I}^{2}}+\frac{2}{\bar{\sigma}_{g}^{2}}\right) \bar{u}_{1}^{2}-\frac{4}{\bar{\sigma}_{g}^{2}} \bar{u}_{1} \bar{v}+\left(\frac{1}{\bar{\sigma}_{I}^{2}}+\frac{2}{\bar{\sigma}_{g}^{2}}\right) \bar{v}^{2}\right]\right\} \\
& \stackrel{\bar{\sigma}_{g} \rightarrow 0}{\rightarrow} \frac{4 \pi G_{0}}{\bar{f}^{1 / 2} \bar{\xi}^{1 / 2}} \int d \bar{v} H(\bar{v}) \delta\left(\bar{u}_{1}-\bar{v}\right)=\frac{4 \pi G_{0}}{\bar{f}^{1 / 2} \bar{\xi}^{1 / 2}} H\left(\bar{u}_{1}\right), \\
& \left\langle I\left(u_{2}=0\right)\right\rangle=\frac{4 \pi G_{0}}{\bar{f} \bar{\xi}^{1 / 2}} \iint d \bar{v}_{1} d \bar{v}_{2} H\left(\bar{v}_{1}\right) H^{*}\left(\bar{v}_{2}\right) \exp \left(-\frac{i \pi}{\bar{z}_{1}} \bar{v}_{2}^{2}+\frac{i \pi}{\bar{z}_{1}^{2}} \bar{v}_{1}^{2}\right) \\
& \times \exp \left\{-\frac{4 \pi^{2}\left[\left(\bar{\sigma}_{g}^{2}+2 \bar{\sigma}_{I}^{2}\right) \bar{v}_{1}^{2}-4 \bar{\sigma}_{I}^{2} \bar{v}_{1} \bar{v}_{2}+\left(\bar{\sigma}_{g}^{2}+2 \bar{\sigma}_{I}^{2}\right) \bar{v}_{2}^{2}\right]}{\bar{\sigma}_{g}^{2} \bar{\sigma}_{I}^{2} \bar{\xi}}+\frac{\left.i 16 \pi^{3}\left(\bar{v}_{2}^{2}-\bar{v}_{1}^{2}\right)\right]}{\bar{\xi}_{\bar{z}_{1}}}\right\} \\
& \stackrel{\bar{z} / \bar{\sigma}_{I} \rightarrow 0}{\rightarrow} \frac{4 \pi G_{0}}{\bar{f} \bar{\xi}^{1 / 2}} \iint d \bar{v}_{1} d \bar{v}_{2} H\left(\bar{v}_{1}\right) H^{*}\left(\bar{v}_{2}\right) \exp \left\{-\frac{1}{4}\left[\left(\frac{1}{\bar{\sigma}_{I}^{2}}+\frac{2}{\bar{\sigma}_{g}^{2}}\right) \bar{v}_{1}^{2}-\frac{4}{\bar{\sigma}_{g}^{2}} \bar{v}_{1} \bar{v}_{2}+\left(\frac{1}{\bar{\sigma}_{I}^{2}}+\frac{2}{\bar{\sigma}_{g}^{2}}\right) \bar{v}_{2}^{2}\right]\right\} \\
& \stackrel{\bar{\sigma}_{g} \rightarrow 0}{\rightarrow} \frac{4 \pi G_{0}}{\bar{f} \bar{\xi}^{1 / 2}} \iint d \bar{v}_{1} d \bar{v}_{2} H\left(\bar{v}_{1}\right) H^{*}\left(\bar{v}_{2}\right) \delta\left(\bar{v}_{1}-\bar{v}_{2}\right)=\frac{4 \pi G_{0}}{\overline{f \xi^{1 / 2}}} \int d \bar{v}_{1}\left|H\left(\bar{v}_{1}\right)\right|^{2} .
\end{aligned}
$$

We can also rewrite the intensity $\left\langle I\left(\bar{u}_{1}\right)\right\rangle$ into

$$
\left\langle I\left(\bar{u}_{1}\right)\right\rangle=\frac{4 \pi G_{0}}{\bar{\xi}^{1 / 2}} \exp \left[-\frac{8 \pi^{2} \bar{u}_{1}^{2}}{\bar{\sigma}_{I}^{2} \bar{\xi}}\right]^{\bar{\sigma}_{g} \rightarrow 0 \text { and } \bar{z} / \bar{\sigma}_{I} \ll 1} \stackrel{4 \pi G_{0}}{\longrightarrow} \frac{\bar{\xi}^{1 / 2}}{\longrightarrow} .
$$

Using Eqs. (A5)-(A7), we can obtain Eq. (12).

[1] M. Schirber, Phys. Rev. Focus 21, 14 (2008).

[2] R. Meyers, K. Deacon, and Y. H. Shih, Phys, Rev. A 77, 041801 (2008).

[3] B. I. Erkmen and J. H. Shapiro, Phys. Rev. A 77, 043809 (2008).

[4] A. Gatti, M. Bondani, L. A. Lugiato, M. G. A. Paris, and C. Fabre, Phys. Rev. Lett. 98, 039301 (2007).

[5] G. Scarcelli, V. Berardi, and Y. H. Shih, Phys. Rev. Lett. 96, 063602 (2006).

[6] T. B. Pittman, Y. H. Shih, D. V. Strekalov, and A. V. Sergienko, Phys. Rev. A 52, R3429 (1995).

[7] A. Gatti, E. Brambilla, M. Bache, and L. A. Lugiato, Phys. Rev. Lett. 93, 093602 (2004); Phys. Rev. A 70, 013802 (2004); J. Cheng and S. Han, Phys. Rev. Lett. 92, 093903 (2004).

[8] Y. Cai and S. Y. Zhu, Opt. Lett. 29, 2716 (2004).

[9] A. Valencia, G. Scarcelli, M. D’Angelo, and Y. H. Shih, Phys. Rev. Lett. 94, 063601 (2005).

[10] F. Ferri, D. Magatti, A. Gatti, M. Bache, E. Brambilla, and L.
A. Lugiato, Phys. Rev. Lett. 94, 183602 (2005).

[11] D. Zhang, Y. H. Zhai, L. A. Wu, and X. H. Chen, Opt. Lett. 30, 2354 (2005).

[12] D. Z. Cao, J. Xiong, and K. Wang, Phys. Rev. A 71, 013801 (2005); M. D'Angelo, A. Valencia, M. H. Rubin and Y. Shih, ibid. 72, 013810 (2005); K. Wang and D. Z. Cao, ibid. 70, 041801(R) (2004).

[13] Y. Cai and S. Y. Zhu, Phys. Rev. E 71, 056607 (2005).

[14] R. Hanbury Brown and R. Q. Twiss, Nature (London) 177, 27 (1956); 178, 1046 (1956); 178, 1447 (1956); R. Hanbury Brown, Intensity Interferometer (Taylor \& Francis, London, 1974).

[15] L. Mandel and E. Wolf, Optical Coherence and Quantum Optics (Cambridge, New York, 1995), Chaps. 5 and 13.

[16] C. L. Mehta and E. Wolf, Phys. Rev. 161, 1328 (1967); 134, A1143 (1964).

[17] J. T. Foley and M. S. Zubairy, Opt. Commun. 26, 297 (1978).

[18] M. Giglio, M. Carpineti, A. Vailati, and D. Brogioli, Appl. Opt. 40, 4036 (2001). 
[19] R. Cerbino, Phys. Rev. A 75, 053815 (2007).

[20] A. Gatti, M. Bache, D. Magatti, E. Brambilla, F. Ferri, and L. A. Lugiato, J. Mod. Opt. 53, 739 (2006).

[21] A. Gatti, E. Brambilla, and L. A. Lugiato, in Progress in Op- tics, edited by E. Wolf (Elsevier, Amsterdam, 2008), Vol. 51, p. 251.

[22] M. Bache, D. Magatti, F. Ferri, A. Gatti, E. Brambilla, and L. A. Lugiato, Phys. Rev. A 73, 053802 (2006). 